decreased oral intake. The child was referred to a tertiary centre for review. On physical examination the child was alert but lethargic with tachycardia to 164 and a fever of 38.9 degrees. She had no drooling, increased work of breathing or stridor. Auscultation revealed equal air entry, normal heart and bowel sounds. Abdomen was soft, non tender without distension. She was treated with antipyretics and chest and abdominal imaging was arranged.

AP chest radiograph and plain abdominal images revealed a circular $2 \mathrm{~cm}$ radiopaque object located in the stomach. On review by radiology the object appeared to have a 'halo sign', a feature consistent with button batteries. Given the uncertain origin of the object and potential for complications associated with button battery ingestion our patient was fasted for an endoscopy.

Endoscopy revealed two 10 cent coins sitting in the gastric antrum with localized erosions and erythema. These were successfully removed with a Roth net and the patient was discharged the same day.

Foreign body (FB) ingestion/inhalation most commonly occurs in children aged between six months and three years. The most common objects are coins, reported in up to $88 \%$ of cases. (1) FB ingestion usually only requires imaging followed by clinical observation. Coins are unlikely to cause complications, whereas button batteries pose a risk of metal poisoning, burns, oesophgeal strictures, perforation, tracheooesophgeal and aorto-oesophgeal fistula and haemorrhage.(2) One study revealed that $14 \%$ of children with oesophageal FBs were asymptomatic on presentation, highlighting the need for imaging. Recommended investigations include AP and lateral neck, AP and lateral chest and abdominal radiographs. (4)

The sensitivity of plan films in detecting a battery was $80.4 \%$, specificity $79.1 \%$ with an overall accuracy of $79.8 \%$. The same study demonstrated that stacking two coins on top of each other lead to high rates of misidentification as batteries with an overall accuracy below 60\%. (1) Importantly this study used artificially produced images which therefore did not include soft tissues and bony structures which could prevent accurate identification.

This case highlights the importance of accurate identification of foreign objects and the need for high clinical suspicion of FB.

\section{P339 THROMBOCYTOPENIA IN CHILDREN WITH FEBRILE ILLNESS: CLINICAL COURSE AND OUTCOME}

${ }^{1}$ Ourania Alexandropoulou*, Lydia Kossiva ${ }^{2}$, ${ }^{3}$ Maria Giannaki, ${ }^{2}$ Maria Tsolia, ${ }^{2}$ Kyriaki Karavanaki. ' General Hospital, Agrinio, Greece; ${ }^{2}$ 2nd Department of Paediatrics, University of Athens, 'PandA Kyriakou' Children's Hospital, Athens, Greece; ${ }^{3}$ Microbiology Department, 'Aghia Sophia' Children's Hospital, Athens, Greece

10.1136/archdischild-2019-epa.688

Background Acquired thrombocytopenia in previously healthy children is common in paediatric practice and usually appears during infections.

Aim To assess the frequency, duration and clinical outcome of postinfectious thrombocytopenia in hospitalized febrile children.

Material-methods 117 febrile children with any kind of cytopenia aged $4.0 \pm 3.8$ years (range $0-14$ ), were admitted to a paediatric department during a 2-year period and were evaluated using inflammatory indices, cultures of body fluids and serological tests.
Results In 52/117 (44.4\%)cases a viral agent was identified. Among them 11/52 (21.1\%) had isolated thrombocytopenia, 32/52(61.5\%) had neutropenia/leukopenia and 9 $(17.3 \%)$ had thrombocytopenia with neutro/leukopenia or anemia.

The patients with viral infection had a mean $( \pm S D)$ age:3.3 \pm 3.9 years. The mean \pm SD duration of fever was $3.4 \pm 2.8$ days.

The highest prevalence of cytopenia was observed during spring (27/52) (51.9\%) and the lowest during fall (13.5\%).

Among the cases with thrombocytopenia, a viral agent was identified in $38.5 \%$ and a bacterial agent in $38.5 \%$.

In children with thrombocytopenia, the most frequently detected viruses were Epstein-Barr virus (10.2\%), followed by a herpesvirus $(6.1 \%)$. In cases with severe thrombocytopenia, mycoplasma infection was most frequent (4.1\%). Finally, in cases with bilineage cytopenia, influenza type A and B were more frequently detected among viruses, and Pseudomonas, Brucella and Rickettsia conorii among bacteria.

In cases of viral infections, thrombocytopenia was transient and lasted for $31.3 \pm 65.5$ days, while in those with 2 cell lines involvement (thrombocytopenia with neutropenia/leukopenia) it lasted for $26.8 \pm 38.9$ days.

In 13 cases with a bacterial infection $2 / 13$ (15.4\%) had thrombocytopenia and $3 / 13$ (23.1\%) thrombocytopenia with neutro/leukopenia.

Among the 21 cases with chronic cytopenia (11 girls and 10 boys), thrombocytopenia was present at 7/21 (33.3\%). Among them an infectious agent was identified in $2 / 7(28.6 \%)$ cases. One child with chronic thrombocytopenia was diagnosed having SLE.

In three children (two with thrombocytopenia and anemia and one with neutropenia/leukopenia and anemia), malignancy was finally diagnosed in a time-period of 4-7 months after the initial assessment. ( 2 with ALL and 1 with MDS).

Conclusion Postinfectious thrombocytopenia in childhood is usually transient with benign course, it resolves spontaneously and is usually associated with common viral or bacterial infections. However, in cases of chronic thrombocytopenia, especially if more than one cell lines are affected, close follow-up is necessary for the early detection of chronic hematologic or autoimmune disease.

\section{P340 CASE REPORT: IMMUNE THROMBOCYTOPENIA PURPURA (ITP) - AN INTRA-ORAL AND EXTRA-ORAL PRESENTATION}

Jennifer Maguire*, Kirsten Fitzgerald, Padraig Fleming. Our Lady's Children's Hospital Crumlin, Dublin, Ireland

10.1136/archdischild-2019-epa.689

A four year-old boy was referred to the Paediatric Dentistry Department by the Haematology Department at Our Lady's Children's Hospital Crumlin. He had a two week history of bleeding and crusting lips and intra-oral ulceration with a history of a cough two weeks prior to this. No relevant family history was noted.

The child's parents had initially presented him to community pharmacy and medical providers who advised attendance at the Emergency Department (ED) of the nearest tertiary care paediatric hospital. Upon presentation to the ED, he had a full blood count performed as part of his work up. Initial 
blood results revealed platelet count of 1 . The rest of his blood results were essentially normal.

On examination, extraoral facial, limb and trunk bruising were noted. Lips were crusted with blood and surrounded petechiae. Intraoral examination revealed soft palate petechiae, areas of ulceration/petechiae in the left and right buccal mucosa and a minor bleed from the lower left lateral primary incisor. The findings were consistent with a diagnosis of Immune Thrombocytopenia Purpura (ITP) precipitated by a viral infection. Initial treatment was with tranexamic acid for five days. On review, there was a marked improvement noted with only minimal blood crusting of the lower lip and fewer intraoral petechiae evident. No further treatment has been required to date. The child is being monitored by haematology and dental specialists with regard to signs, symptoms and progress.

ITP is a haematological disorder defined by low platelet count. Children often develop this following a viral infection. Spontaneous bleeding or bleeding induced by trauma, may be the first clinical signs of ITP. This interesting case highlights a primary presentation of ITP with oral signs. This situation may present to a General Dental Practitioner or General Medical Practitioner in community or another Healthcare Professional and it is important to recognise the signs and symptoms and intervene appropriately

\section{P341 REVIEW OF PAEDIATRIC PATIENTS WITH SEVERE FACTOR $\times$ DEFICIENCY IN IRELAND}

Mary Kavanagh*, Imelda Kelly, Caitriona Ferry, Bridin Brady, Irene Regan, Beatrice Nolan. OLCHC, Dublin, Ireland

\subsection{6/archdischild-2019-epa.690}

Severe Factor $\times(\mathrm{FX})$ deficiency is a rare autosomal recessive bleeding disorder. Patients with FX:C level of $<0.01-0.03$ $\mathrm{iu} / \mathrm{ml}$ have a severe bleeding phenotype with haemarthrosis occurring in $69 \%$ of patients and intracranial haemorrhage accounting for $15 \%$ of all bleeding events. Prophylactic FX replacement is challenging and usually requires a central venous access device (CVAD). This review included 4 paediatric patients with severe FX deficiency, aged between 1 and 16 years. Three were born to consanguineous parents. Factor $\times$ mutations have been identified in all patients. $2 / 4$ of this patient cohort were diagnosed at birth due to a previously identified family history, $1 / 4$ was diagnosed at day three of life when he presented with an intracranial haemorrhage and $1 / 4$ presented at day three of life with epistaxis. All 4 patients commenced Prothrombin Complex Concentrate (PCC) prophylaxis in the first week of life. Dosing regimens range from 25 - 60 units per kilogram once to twice weekly to maintain trough factor $\times$ level $>$ $0.05 \mathrm{iu} / \mathrm{ml}$. There have been no spontaneous life or function threatening bleeding episodes while on prophylaxis. All patients had CVAD inserted with PCC support. Three patients had CVAD removed because of Staph aureus septicaemia. One of these three patients developed CVAD related iliofemoral thrombosis, necessitating anticoagulation. In conclusion, with early recognition and diagnosis of severe FX deficiency, bleeding symptoms can be effectively treated and managed.

\section{P342} CLINICAL COURSE IN CHILDREN DIAGNOSED WITH HEREDITARY SPHEROCYTOSIS IN ALBANIA

Gladiola Hoxha*, Donjeta Bali, Mirzana Kapllanaj, Mirela Xhafa, Elizana Petrela, Anila Godo. University Hospital Center 'Mother Teresa', Tirana, Albania

\subsection{6/archdischild-2019-epa.691}

Objective To describe the clinical spectrum of children diagnosed during 15 years with hereditary spherocytosis, classify them on 4 groups based on their clinical data and evaluating their outcome and giving our recommendations for their management.

Methods This is a analytic, observational, retrospective and case-control study analyses of 30 children diagnosed and followed up at our center during 15 years (2003-2018). The patient were diagnosed with hereditary spherocytosis based on their clinical history, clinical examinations, and on a positive osmotic fragility curve. They were divided based on the clinical form on 4 groups : mild, moderate, moderate to severe and severe group. The groups were compared between them regarding the laboratory data, the need for transfusion, cholelithiasis, splenic sequestration, aplastic crises and splenectomy.

Results In our study 3 patients had mild H.S (10\%), 10 patients had moderate H.S (33.3\%), 10 patients had moderate to severe H.S (33.3\%) and 7 patients had severe H. S (23.4\%). The mean age at diagnosis was 4.7 years Patient with severe form were younger than patients with moderate form $(p=0.016)$ and had lower hemoglobin level $(p \leq 0.001)$, higher reticulocyte count $(p=0.049)$ and also needed more transfusions $(p=0.002)$. There was not found a correlation between the level of bilirubin and clinical gravity $(p=0.873)$. Splenic sequestration was commonly found, $63.3 \%$ of patiens experienced a splenic sequestration and its frequency was significant higher in severe patients $(p=0.011)$. Cholelithiasis was present in half of the patients but its development was not related with disease's severity $(p=0.391)$. Aplastic crisis was relatively rare, only one patient developed aplastic crisis and it was due to parvovirus infection. Splenectomy was performed in 16 patients $(53.3 \%)$ with the main indication transfusion dependence and splenic sequestration.

Conclusion We have a high number of children with severe clinical forms because as they do severe forms they came at our center and are diagnosed compare with mild forms which are asymptomatic and remain undiagnosed. The clinical course of our patients was relatively benign, but we still have a high frequency of splenic sequestration $63.3 \%$ (most of them due to recurrent infections) a high rate of blood transfusion (54\%) and a high number of splenectomised patients.

\section{P344 A RARE ASSOCIATION: ACUTE DISSEMINATED ENCEPHALOMYELITIS IN CHILD AFFECT BY AUTOIMMUNE HAEMOLYTIC ANAEMIA AND AUTOIMMUNE THROMBOCYTOPENIA}

${ }^{1}$ Lucia Soldano*, ${ }^{1}$ Francesca Romana Consiglio, ${ }^{1}$ Anthea Bottoni, ${ }^{1}$ Teresa Merola, ${ }^{2}$ Angela Maggio, ${ }^{2}$ Raffaela De Santis, ${ }^{2}$ Anita Spirito, ${ }^{2}$ Saverio Ladogana, ${ }^{3}$ Massimo Pettoello-Mantovani. 'Pediatric Residency Program, Foggia, Italy; ${ }^{2}$ Departement of Paediatric Onco-Haematology, IRCCS Casa Sollievo Della Sofferenza, San Giovanni Rotondo, Foggia, Italy; ${ }^{3}$ Departement of Paediatric, IRCCS Casa Sollievo Della Sofferenza, San Giovanni Rotondo, Foggia, Italy

10.1136/archdischild-2019-epa.692

Acute disseminated encephalomyelitis (ADEM) is an inflammatory demyelinating illness, characterize-ed by a 